\title{
Time for new guidelines in advanced diabetes care: Paradigm change from delayed interventional approach to predictive, preventive \& personalized medicine
}

\author{
Olga Golubnitschaja
}

Received: 8 January 2010 / Accepted: 11 February 2010 /Published online: 16 March 2010

(C) European Association for Predictive, Preventive and Personalised Medicine 2010

\begin{abstract}
Global burden of diabetes mellitus clearly demonstrate inadequacy of current diabetes care measures: the costs of caring for patients with diabetes mellitus are the highest compared to other frequent pathologies. Nonetheless, for every $10 \mathrm{~s}$ one patient dies of diabetes-related consequences. Thus, there is urgent need for highly effective measures that would lead to reduced prevalence, better longterm outcomes and improved quality of life for diabetic patients reducing associated economic burden. Such targeted measures would require the creation of new guidelines for advanced diabetes care that would provide for regulation, for timely predictive diagnostics as well as an effective prevention and creation of individualized treatment algorithms. Effective communication among the research community healthcare providers, policy-makers, educators and organized patient groups (Federations of Diabetics) is of paramount importance and essential for (pre)diabetes care. The ultimate mission of the "European Association for Predictive, Preventive \& Personalised Medicine" is to promote this process in Europe and across the globe.
\end{abstract}

Keywords Healthcare providers · Strategic trends ·

Advanced technologies · Navigating diagnostic systems .

Socioeconomical aspects $\cdot$ Education

O. Golubnitschaja

"European Association for Predictive,

Preventive \& Personalised Medicine",

Bonn, Germany

O. Golubnitschaja $(\square)$

Department of Radiology,

Rheinische Friedrich-Wilhelms-University of Bonn,

Sigmund-Freud-Str. 25,

53105 Bonn, Germany

e-mail: Olga.Golubnitschaja@ukb.uni-bonn.de

URL: www.epmanet.eu
Predictive diagnostics as the platform for personalized medicine: new philosophy \& strategic trends in healthcare

Predictive, preventive \& personalized medicine is a new philosophy in healthcare aimed at application of innovative biotechnologies in the prediction of human pathologies, the development of timely prevention and individualized therapy-planning. The advantages of predictive diagnostics and personalized treatment include prevention of the majority of disorders, personalized treatment, individual therapy planning, substantial improvement of life-quality, plausible solutions for particular social and ethical and serious economical problems that we face in the current healthcare system. This kind of progress can be achieved by highly professional application of currently available biotechnological approaches focused on solving the accumulating problems in healthcare.

Predictive diagnostics is considered as a reliable navigation system for targeted preventive measures and consequent development of individualized treatment approaches. Of paramount importance is communication among professionals - medical doctors, biotechnologists, computerscientists, healthcare providers, policy-makers, educators, who are obligatorily involved in the paradigm change from curative (interventional) to predictive medicine. This concept is considered as medicine of future. The paradigm change can be achieved only by well-coordinated fulfillment of the following components:

- adequate investment creating novel technologies

- development of non- or minimally-invasive diagnostic tools

- well-organized process for exchange and transfer of knowledge among biomedical research entities and biotechnological industries for production of the advanced diagnostic tools 
- quality assurance through the introduction of international standards for technological tools and devices, patenting and licenses

- appropriate professional education in terms of the application of high-tech biotechnology in medicine

- effective regulations in the healthcare sector: introduction of the obligatory guidelines and clear regulations for the health insurance industry to ensure patients needs are met

- measures to ensure confidentiality of patient information and personal databank

- distribution of relevant information among healthcare professionals and users.

The overall concept of the paradigm change to predictive and personalized medicine has been recently presented in the worldwide pioneer book worked out by 60 leading experts from 16 countries [1]. The coordinated measures, described above, should be well-focused on solving the accumulating problems in healthcare and the concomitant economical burden that societies are increasingly faced with across the globe.

\section{The mission of the European Coordinator in this field is performed by the "European Association for Predictive, Preventive and Personalised Medicine"}

The concepts of innovative European and international projects, which EPMA represents for further consideration at the EU-Commission, the European Parliament, WHO and UNO (see Fig. 1) are worked out by the consortium of the world-leading professionals (Europe-unrestricted).

The first meeting of the EPMA-Representatives with the Vice-Secretary General of UNO took place in Geneva on December 8th 2009. The EPMA-Mission and-Objectives in the field of Predictive, Preventive \& Personalized Medicine (PPPM) have been introduced to the Vice-Secretary General of UNO. The participants of the meeting agreed that the paradigm change from curative to PPPM can be achieved only by coordinated measures well-focused on solving the accumulating problems in healthcare and the concomitant economical burden that societies across the globe are facing more and more. This is a new philosophy in healthcare and the platform for personalized patient's treatment considered as medicine of future.

Practical application of innovative technologies in favor of predictive diagnostics, targeted preventive measures and personalized patient treatment in European healthcare is the central idea of the Association. By the international EPMAJournal, the Association welcomes a professional forum to discuss most effective technologies and innovative

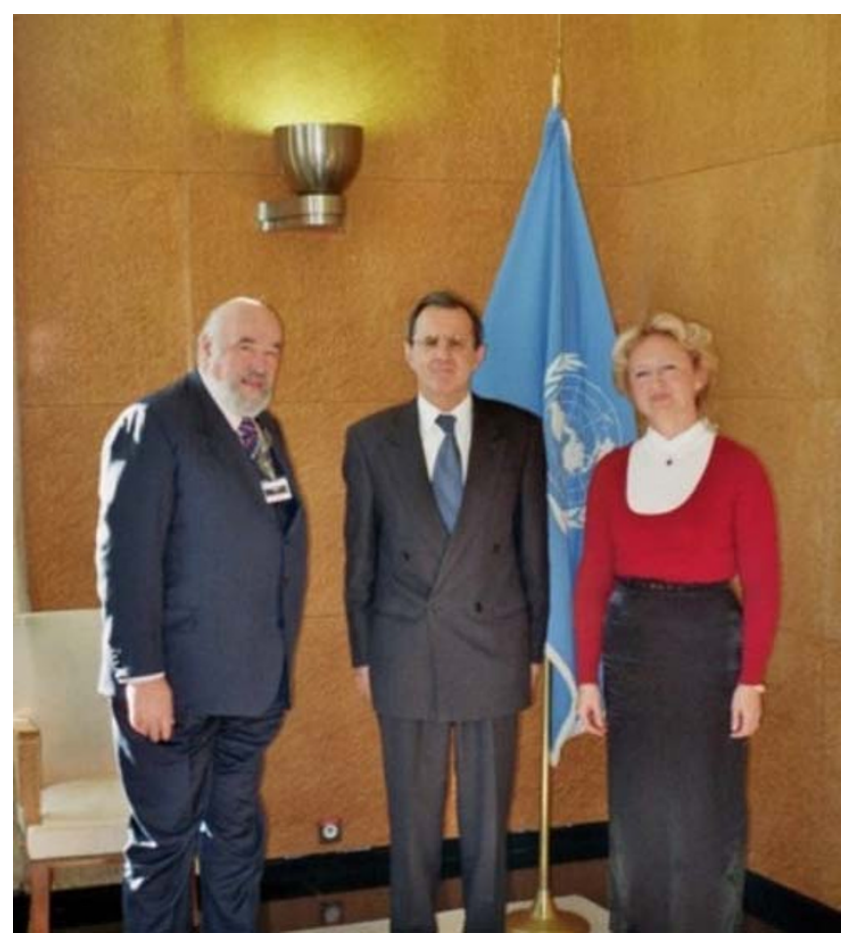

Fig. 1 EPMA goes global in consensus with United Nations. From left to right: EPMA-President-Vincenzo Costigliola, UNO-Vice-Secretary General-Sergej Ordzhonikidze, EPMA-Secretary General-Olga Golubnitschaja

approaches of predictive, preventive and personalized medicine.

\section{Advanced (pre)diabetes care as the best model for timely prediction, prevention and personalized treatment}

In terms of the urgent necessity for paradigm change from interventional measures to predictive, preventive $\&$ personalized medicine, one of the best examples is healthcare of permanently growing cohort of diabetic patients, which represents a serious economical problem in most industrialized countries and countries with large populations. Diabetes mellitus (DM) frequently results in diverse severe complications, such as retinopathy, nephropathy, silent ischemia, dementia, and cancer. Diabetes care faces a whole spectrum of problems including the necessity for population screening, targeted preventive measures, ethics, economics, and broad dissemination of the issue-related information that still await a dramatic improvement in each aspect. As recently reviewed [2], at this stage we recognize:

- the epidemic scale of the disease,

- the contemporary onset of the dominant diabetes type 2 in youth or even in childhood, and 
- a permanently growing number of well-acknowledged severe complications of diabetes mellitus

Diabetes mellitus type 2, historically characterized as an adult-onset disorder, now comprises approximately one third of new DM-cases of those already in their second decade of life [3]. Imbalanced nutrition and widespread "fast food" consumption as well as irregular and low physical activity in childhood contribute to the increasing prevalence of obesity in youth. Consequently, the mean age at diagnosis of DM type 2 in young population is $12-$ 14 years as reviewed by Huang \& Goran [4].

Despite the current progress in individualized insulin therapy, the common onset of DM type 2 in early adulthood results in dramatic consequences that are linked to the early onset of diverse severe complications such as retinopathy, nephropathy, silent ischemia, and dementia. Chronic degenerative processes affecting vital organs are wellacknowledged as secondary DM complications. They usually appear as a "domino-effect" in the characteristic sequence as reviewed earlier [5]. Proliferative diabetic retinopathy is attracting much attention being considered as an early indicator of individual predisposition to the cascade of chronic complications responsible for the majority of morbidity and mortality in diabetics.

The recent years have brought new knowledge concerning the predisposition to cancer in diabetic patients: large population studies indicate an increased risk of liver, pancreas, bladder, digestive and urinary tracts, and endometrial cancer in DM with some ethnic and age-specific differences as well as gender-dependent preferences [6, 7]. Although the molecular pathomechanisms of secondary diabetic complications require further investigations, emerging research has contributed well to our understanding of the pathology in its whole complexity. Extensive damage to DNA is well documented for cells isolated from DM-patients [8] and can be well explained by impaired glucose/insulin metabolism. These findings indicate an imbalance between the increased production of ROS and decreased DNA-repair capacity in diabetics. As long as damaged cells can be repaired, the integrity of a corresponding cell population remains intact. However, as soon as a critical number of cells have damaged, two potentially dangerous processes may be triggered in affected organs: depending on the quality of cell-cycle controlling machinery. This imbalance can lead either to extensive apoptotic cell loss or proliferation of damaged cells. Whereas the first process mainly causes tissuedegeneration, the latter predisposes to pre-cancerous lesions and tumor development. Both (neuro)degenerative alterations in damaged organs and predisposition to cancer are well documented in diabetes mellitus [9]. At early stages, those alterations can be monitored by advanced analytical technologies and used as a navigator for targeted preventive measures in the group at high risk.

Given the high risk and prevalence of secondary complications as well as individual predisposition to target organ injury, DM is the best model for the application of predictive diagnostics aimed at preventive measures and personalized treatment. Generally, there are three levels desirable for pre- and diabetes care [2]:

- 1st level: prediction of the predisposition early in childhood

- 2nd level: prediction of early/premature aging and prestages of diabetes

- 3rd level: prediction of diabetes-related complications.

\section{First level-general predisposition to diabetes: postnatal diagnostics, preventive measures early in life, personalized treatment, and education}

There is a growing body of evidence demonstrating certain endogenous and exogenous factors to be strong contributors to the prevalence of DM worldwide. Thus, the influence of economic aspects with the respect to a higher DM-prevalence in developing countries is evident [6, 10]. Further, current population studies in perinatal and postnatal diagnostics clearly show that developing countries have the highest numbers of newborns affected by birth asphyxia - the most frequent cause of mortality in children under 5 years of age as well as of short- and long-term complications worldwide [11]. It is noteworthy that the educational level of parents correlates inversely with high risk and incidence of asphyctic babies born across the population.

What is the link between diabetes and the most frequent perinatal pathology_-birth asphyxia? Molecular biological analysis performed with animal models of perinatal asphyxia provides clear evidence for a high predisposition of asphyctic newborns to severe long-term outcomes such as diabetes mellitus, neurodegenerative, cardiovascular and cancer diseases [12]. Further diabetogenic effects have been demonstrated for (a)typical antipsychotics (e.g. methylphenidateinhibitor of serotonin-reuptake) which are frequently prescribed to treat attention-deficit / hyperactivity disorder (ADHD) in children and youth [13, 14]. This disorder is one of the long-term outcomes of birth asphyxia. In particular cases, new onset of DM type 2 was observed within first few days under ADHS-treatment with the (a) typical antipsychotics $[15,16]$.

How can this knowledge be used in favor of targeted preventive measures? Parents should be educated about the risk factors and potential long-term outcomes, in order to possibly prevent asphyxia. In the case of the asphyxiated newborn, complementary postnatal diagnostic approaches 
should be applied to estimate the degree of the organdamage and potential long-term outcomes. Promising noninvasive blood-tests are currently under development which would allow a more precise diagnosis and reliable prognosis for severe pathologies (diabetes, cancer, etc.), which can be potentially developed later in life [12]. Further educational measures should consider well-known pathology-relevant exogenous and endogenous factors such as environmental aspects, genetic background, nutrition, life style, stress, infections, job- and physical-activities [10, 17-19]. Some of the central factors and consequent recommendations have been recently reviewed [2]. Physicians should be well-educated for medication with antipsychotics: it is advised to be cautious with the prescription of diabetogenic agents [15]. Particular care is required when treating ADHD in childhood and youth: special attention should be paid to high-risk patients predisposed to onset of DM. Monitoring of DM-relevant parameters (such as dynamic of body-mass-index and blood-glucose levels) is advised for careful set-up of individualized treatment approaches.

\section{Second level: Adulthood and pre-stages of Diabetes mellitus}

Aging is one of the strongest contributors to the development of the majority of human pathologies including DM. In particular, early or even premature aging is especially of concern, since this supports or even triggers the (pre) lesions, pre-stages and the consequent pathologies early in life. Early aging can have diverse origins and usually results from the interaction of genetic, epigenetic and various environmental factors. The well-known causes of the early/premature aging are inborn genetic disorders, such as Down Syndrome, deregulation of longevity-associated genes, disconnected functional interaction between nuclear and mitochondrial genomes, environmental factors and inappropriate reaction towards any kind of stress. The common resulting effect is an imbalance in the production of aggressive reactive species, amounts of antioxidants and the activity of detoxification pathways that altogether support extensive organ-damage [20].

The activity of detoxification pathways consistently decreases for everybody during life. However, this activity-fall is highly individual and can be estimated noninvasively by non-sophisticated in vitro tests measuring the induction of SOD-activity towards stress-conditions triggered, e.g. by the in vitro application of paraquat to leukocytes. The results of the test support well the keyrole of early aging in the manifestation of major pathologies such as DM type 2 [21]. As reviewed earlier [2] the most important message considering the targeted preventive measures against early aging and manifestation of diabetic pre-stages is the following:

1. Individuals within the 5th, 6th and 7th life-decades demonstrate the biggest diversity in the induction of the stress-reaction. These are the groups of particular interest for examination and targeted prevention of early aging.

2. Some individuals in the 6th decade of life exhibit parameters similar to those in the 4th and even 3rd ones; others, however, tend to show parameters more characteristic for the 9th and 10th decades of life. The latter are at high risk for aging-related pathologies. For specific predispositions should be looked by predictive diagnostic tools.

3. The individuals in the 10th decade of life demonstrate better values compared to the majority in the 8th decade. This group may provide us with new knowledge about particularities of metabolism well protected against aging.

An application of tests aimed at prediction of accelerated aging is not expensive but very useful for the prediction of aging-related pathologies such as diabetes mellitus.

Recent innovative diabetes-related studies offer a more precise definition of the diabetic phenotype at the molecular level that may prove useful in delineating diabetogenic genes or gene products in diabetes pre-stages: an identification of secreted and pancreas specific proteins/peptides responsible for abnormalities of insulin secretion in type 2 $\mathrm{DM}$ and which might serve as predictive markers for $\beta$-cell dysfunction in diabetes pre-stages was performed [22]. The analysis resulted in the identification of 102 secreted proteins which can be divided into five classes: (a) hormones and related molecules, (b) protease inhibitors, (c) secretory vesicle proteins, (d) cell adhesion or extracellular matrix proteins, and (e) secreted enzymes. From all identified secreted proteins only pancreatic polypeptide Y (PPY) was identified as pancreas specific thereby, fulfilling the criterion of a predictive marker. Further strategies for the detection and identification of additional pancreas specific biomarkers and characteristics for deficiency in insulin secretion are necessary. Since one ideal biomarker is difficult to find or may even not exist at all, a potential solution might be to use the combinatorial power of several biomarkers, each of which alone may not offer satisfactory specificity and sensitivity. The creation of individualized care algorithms is closely linked to the identification of a set of molecular-targets that would serve as the basis for the early diagnosis of pre-diabetes, therapy selection and disease monitoring that together could lead to personalized treatment options [23].

Currently, for individuals highly predisposed to the early aging / extensive aging-processes generally and, in particular 
to diabetes pre-stages, the following preventive and therapeutic measures are recommended:

- The life-style should be personally discussed with the specialists to develop individualized nutrition and optimized physical and further activity taking into account all related stress contributors including the relevant environmental factors [24];

- Targeted metabolic corrections might be performed [5];

- Gene-therapy and alternative forms of individualized therapy approaches can be potentially applied [25].

\section{Third level: Prediction and personalized treatment approaches slowing down or even preventing severe complications secondary to diabetes}

Chronic vasodegenerative disease as early manifestation of the complication-cascade

In addition to the aging processes, there is the likelihood of extensive damage to numerous organs in diabetes. Severe complications that develop secondarily to DM represent the main cause of morbidity and mortality among diabetic patients. According to the current worldwide statistics, DM is currently the fourth leading cause of death [26], in particular, due to fatal DM-related complications [27]. Since our knowledge concerning all DM-triggered secondary pathologies is currently incomplete, the real figures for DM-related mortality are assumed to be significantly higher than considered till now. The common onset of DM type 2 in early adulthood leads to dramatic consequences linked with the early onset of diverse severe complications such as retinopathy, nephropathy, silent ischemia, dementia and "diabetic foot". Chronic degenerative processes affecting vital organs are well acknowledged in DM; they usually appear as a "domino-effect" in the characteristic sequence reviewed earlier [5]. An active proliferative diabetic retinopathy is considered to be an early indicator of an individual predisposition to the cascade of severe chronic complications that usually develop down-stream towards the manifestation of DRP. Therefore, early/predictive diagnosis of DRP is a reasonable indication for immediate preventive measures and individualized therapy [28]. Diabetic angiopathy involving the small capillary vessels and intra-retinal fluid collection in macula can be the earliest signs of DM, even before the diagnosis of an "active" disease. Consequently, the retinal screening in the risk subpopulations makes good sense to be performed, before other manifestations are noted. Moreover, the early ischemic alterations in the retina can predict the prognosis and even the severity of possible systemic complications to be expected. Retinal ischemic changes detected by Fluorescein Angiography are the reliable indicator for systemic ischemic changes followed by blood flow disturbances leading to ischemic brain and diabetic foot.

Diabetic Poly-Neuropathy (DPN)

Since the topic "Predictive, Preventive \& Personalized Medicine in treatment of Neurodegenerative Diseases" will be regularly discussed in the special issue of the EPMAjournal, here we provide only a brief overview of important aspects of DPN:

$\triangleright$ DPN is the most common form of peripheral neuropathy accompanied by sensory loss, visual loss, compromised pain sensation and brain atrophy [29-31]. $>$ Retinopathy is developed by $30-50 \%$ of diabetic patients [28].

$\gg$ Extremely high co-morbidity is observed for patients with sight-threatening diabetic retinopathy [32].

$>$ Early retinopathy is the reliable indicator of severe diabetic complications upstream of the cascade of degenerative processes in other organs. Consequently, proliferative diabetic retinopathy is frequently accompanied by diabetic maculopathy, angina, painless cardiac infarction, stroke, renal disease, foot ulceration [33-36]. $>$ Following relevance of DPN is considered for the major neurodegenerative pathologies:

- Both DM type 1 and Multiple Sclerosis are autoimmune disorders with etiological similarities [37].

- The common feature of DM and Parkinson's Disease is the mitochondrial dysfunction [38, 39].

- DM is of limited relevance for the development of Alzheimer Disease, which is considered as Diabetes type 3 due to insulin resistance in particular organ - the brain [40-42].

- Diabetes is considered of no special risk for glaucoma pathology [43].

Cancer predisposition in Diabetes: contributing factors and indicators to predict the pathology

The recent years have brought new knowledge concerning the predisposition to cancer in diabetic patients: the large population studies performed indicate an increased risk for and mortality related to liver, pancreas, bladder, kidney, colon, stomach, rectum, endometrium, ovarian, and cervix cancer types in DM with some ethnic and age-specific differences as well as gender-dependent preferences [6, 7]. The etiology of cancer in DM remains elusive. However, it is evident that multiple factors contribute to cancer in DM including the following:

- Major stress factors (excessive metabolic alterations, disturbed glucose/insulin homeostasis, hormonal deregu- 
lation, insufficient detoxification) with consequent excessive production of ROS.

- Mitochondrial dysfunction with consequent low energy production and insufficient repair capacity, accumulating damage to both mitochondrial and chromosomal DNA.

- High risk for infectious disorders with consequently induced viral pro-oncogenic activity as well as activity of particular pathogenic bacterial forms such as Helicobacter pylori.

Cancer predisposition in Diabetes is reviewed in this journal-issue [44].

Advanced diagnostic approaches for prediction of secondary diabetic complications

A prime target of hyperglycemia-induced injury is the endothelial cells, the damage of which contributes to the macro- and microvascular complications. Consequently, cardiovascular diseases are the leading cause of death in diabetic patients, accounting for about $65 \%$ of the deaths among patients with type 2 diabetes mellitus; much of the morbidity and mortality relates to atherosclerotic coronary artery disease, congestive heart failure and sudden cardiac arrest [35]. A further significant portion of diabetic patients develop cancer or neurodegenerative diseases as described in the above subchapters. Importantly, however, not all diabetic patients experience severe long-term complications irrespective of blood glucose control. For example, about $20-40 \%$ of diabetic patients ultimately develop a nephropathy but it is not clear why all diabetic patients do not manifest this complication. Nonetheless, differential susceptibility to chronic diabetic complications indicates that, aside from hyperglycemia, other factors (e.g. genetics) modulate an individual's risk for vascular complications. These aspects emphasize the need for an individualized approach to detection and management of diabetes mellitus. As a result, identification of effective diagnostic markers and prognostic indicators unique to diabetes mellitus should prove valuable for early diagnosis and targeted preventive or individualized interventional measures.

Although urinary proteomics is considered as a potentially powerful diagnostic tool for the early detection of diabetic renal damage, major limitations of the technology should be addressed. From a technical viewpoint, the analysis of low level proteins is the major limitation. Therefore, gel-free analytical systems combined with prefractionation prior to analysis are required to solve this technical problem. Further, the examination should be very well designed, particularly in terms of sample collection. Thus, peptide patterns for first-void and mid-stream female urine are different, i.e. a contamination from non-urinary passage is an obvious obstacle, particularly for analyses conducted with female subgroups [45]. Finally, data achieved by urinary proteomics are complicated due to their correct interpretation currently giving rise to many unanswered questions. Since disease specific changes in blood-plasma and vascular proteomes occur up-stream towards the majority of organ systems in type 2 diabetes [46], it should be clarified as to which changes monitored in the renal proteome originate from the kidney and which from other organs. Therefore, the current challenge is whether predictive indications for developing secondary diabetic complications, including nephropathy, should be sought in plasma rather than in urinary proteomics [5]. A detection of pathology-specific circulating nucleic acids in blood-plasma is considered as one of the most potent diagnostic approaches in the individualized treatment of diabetic patients [47, 48]. Thus, reliable prediction of endothelial dysfunction in diabetes basing on circulating markers [49] and prognostic targets specifically for diabetic retinopathy basing on detection of oxidative-stress and apoptotic metabolites in blood is proposed in this journalissue [50].

A non-invasive molecular diagnostic approach based on disease-specific gene expression patterns in circulating leukocytes has been recently suggested for the predictive diagnosis of secondary diabetic complications [51]. This approach is based on disease-and severity-specific alterations in the gene expression patterns of circulating leukocytes and protease activity in serum that can be ex vivo evaluated in individual blood samples. The test foresees a precise expression profiling of selected genes in circulating leukocytes isolated from fresh blood samples. These genes, which have been proposed to play a role in the pathology of type 2 diabetes, belong to the stressproteome and have a function in the following pathways: cell-cell communication, cell-adhesion, apoptosis, tumorigenesis, transcription regulation, tissue remodeling and neurodegeneration. A clinical application of the test is currently under consideration, and a nanotechnology which should provide a possible easy and relatively inexpensive routine application of the test is under development [52].

\section{Creation of individualized therapy approaches}

In light of the above, an early and predictive diagnosis followed by targeted preventive measures and individualized therapy approaches would dramatically improve the overall outcome of secondary complications in diabetic patients. An excellent example can be given by advanced ophthalmo-diabetological care for individualized patient treatment, e.g. when therapeutic approaches such as vitrectomy are applied [53, 54]. The technique of vitrectomy is rapidly improving and clinical guidelines should take 
its progress into consideration. Novel techniques of intravitreal drug application show some positive effects. However, there is a lack of randomized, well-controlled trials that can potentially result in improved guidelines for clinical practice. Severe and moderate vision loss in diabetes mellitus is essentially preventable by advanced early and predictive diagnostics and personalized patients treatment. Advanced treatment approaches are possible only by understanding the molecular and pathophysiological mechanisms of the disease and precise prediction of individual complications. The interplay between three vasoactive systems, VEGF, prostaglandins and NO, have predictive power in the progression of the ischemic retinal changes [39]. As for the individual treatment, the goal of the personalized vitrectomy is accomplished with the modern drug usage such as anti-VEGF therapeutic approaches, the algorithms for which can be personally modeled depending on the kind and stage of diabetic retinopathy. Further, advanced reparative cell-therapy approaches have been suggested to treat vasodegenerative disease in the retina [55].

A further example can be given for the prediction and individualized treatment of aortic valve degeneration and replacement in diabetic patients. On average, $20-30 \%$ of implanted bioprostheses show dysfunction after about 10 years post implantation. Recent reports predict that a greater than $50 \%$ incidence of failure will be seen in bioprostheses at $12-15$ years [56]. These figures are much worse for diabetic patients, who are at high risk for degeneration of both native valvular tissue and bioprostheses [20]. To predict the progressive valvular degeneration and necessity of accelerated re-implantation, comparative expression analyses have been undertaken demonstrating the high predictive power of some marker-candidates and novel therapeutic targets [57, 58]. Among them-the ECMdegrading enzymatic-core, the expression profile of which changes dramatically at the remodeling of valvular geometry and complications such as accelerated degeneration of bioprostheses [36, 58]. Moreover, the activity of the tissueremodeling key-enzymes-metalloproteinases MMP-2 and MMP-9 - can be non-invasively measured in blood by ex vivo zymography [5]. Individual stress response, quality of cell-cycle control, and imbalance in tissue-remodeling protein-complexes may play a pivotal role in severity of degenerative processes. This opens good perspectives for predictive diagnostics, new targets and individualized therapy approaches for diabetic patients [59].

Further, intense research is currently devoted to the detection of pathology-specific molecular pathways for consequent development of improved diagnostic and therapeutic targets: the potential role of Indoleamine-2,3Dioxygenase-dependent molecular pathway as an effective predictive and therapeutic target in diabetes diagnostics and treatment is demonstrated in this journal-issue [60]. For early detection of diabetic nephropathy and consequent monitoring of pathology-progression under individually created treatment algorithms, specifically inflammatory cytokines can be chosen for development of advanced prognostic tools as reviewed by Elmarakby et al [61].

Advanced drug delivery systems present indubitable advances for organ-selective and targeted treatment of diabetes and secondary pathologies. Minimally invasive
Fig. 2 The overall EPMAconcept of the communication and output among field-related researchers, healthcare providers, policy-makers, educators and organized patient groups (e.g. Federations of Diabetics) that are intimately involved in (pre)diabetes care

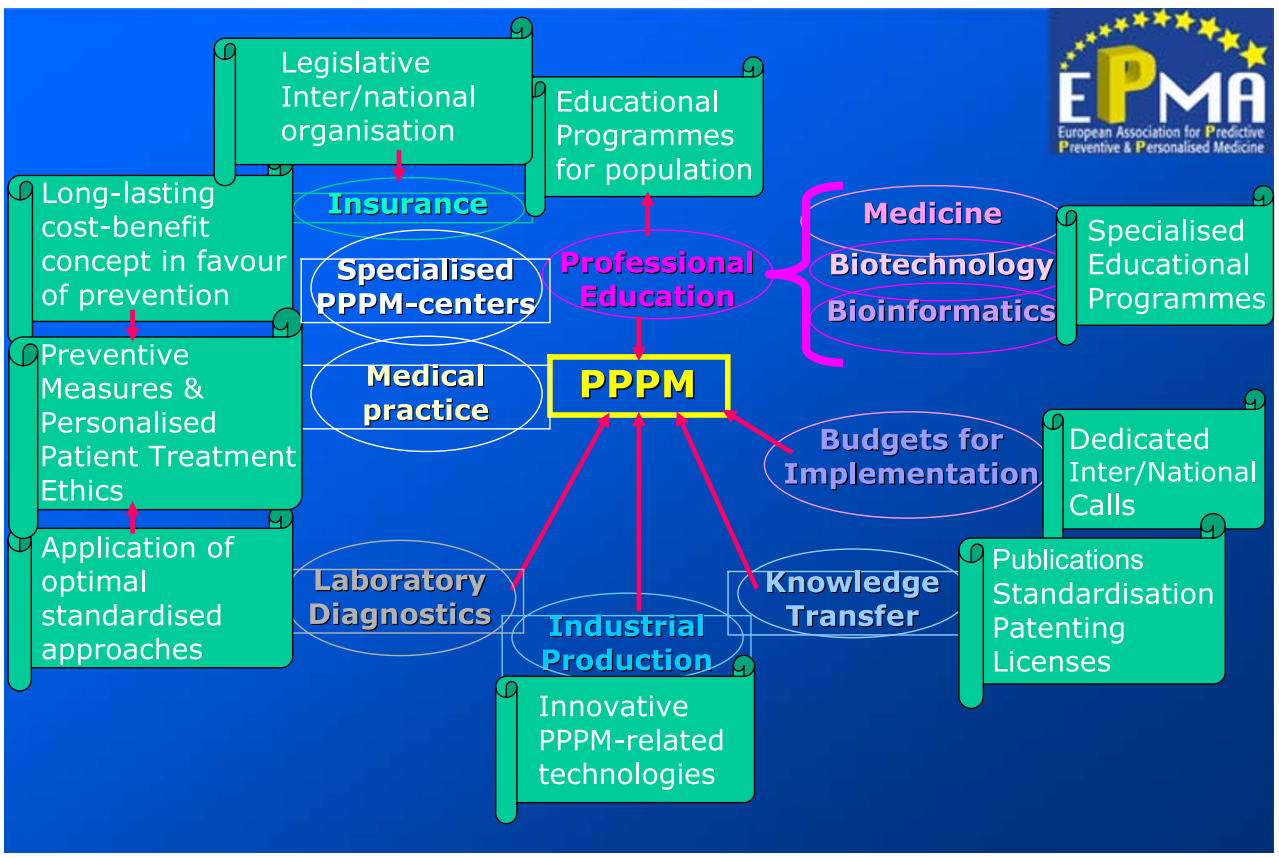


insulin administration approaches and / or improvement of pancreatic insulin secretion is the main goal of novel drug delivery systems and is highly desirable for advanced treatment of diabetic patients with both type 1 and type 2 [62-65].

Noteworthy, using individualized treatment algorithms based on well-established conventional methodology, such as optimized surgical timing in treatment ocular diabetic complications, post-interventional recovery, long-term outcomes and life-quality of treated diabetics can be dramatically improved as reported in this issue [66, 67].

Surprisingly, the highly relevant topic of periodontitis risk in diabetic subpopulation is rarely discussed in context of advanced diabetes care. Current journal-issue promotes this discussion with a contribution from the experts in the field [68].

\section{Conclusions \& outlook}

- Global figures clearly demonstrate inadequacies of current diabetes care: actual economical burden are the highest for diabetes care compared to all other frequent pathologies.

- Yet, every $10 \mathrm{~s}$ one patient dies of diabetes-related consequences.

- Due to epidemic scale of the pathology, severe complications and extremely high co-morbidity, current diabetes care requires urgent and highly effective measures that would lead to reduced prevalence of diabetic complications, better long-term outcomes and life quality of patients as well as reducing the growing economic burden.

- These targeted measures require the creation of new guidelines for advanced diabetes care which would provide for proper regulation of timely predictive diagnostics, an effective prevention and creation of individualized treatment algorithms.

- Communication among scientists, healthcare-providers, policy-makers, educators and organized patient groups (e.g. Federations of Diabetics) intimately involved in (pre) diabetes care is of paramount importance (see Fig. 2).

- Global organizations involved in the process should be well networked in the topic in order to promote this important mission.

\section{References}

1. Costigliola V, Gahan P, Golubnitschaja O. Predictive Medicine as the New Philosophy in Healthcare. In: Golubnitschaja O, editor. Predictive Diagnostics and Personalized Treatment: Dream or Reality? New York: Nova Science Publishers; 2009.
2. Golubnitschaja O. Advanced Diabetes Care: Three Levels of Prediction, Prevention \& Personalized Treatment. Curr Diabetes Rev. 2010;6:42-51.

3. Rosenbloom AL, Joe JR, Young RS, Winter WE. Emerging epidemic of type 2 diabetes in youth. Diabetes Care. 1999;22:345-54.

4. Huang TT, Goran MI. Prevention of type 2 diabetes in young people: a theoretical perspective. Pediatr Diabetes. 2003;4:38-56.

5. Golubnitschaja O. Clinical Proteomics in application to predictive diagnostics and personalized treatment of diabetic patients. Current Proteomics. 2008;5:35-44.

6. Cebioglu M, Schild HH, Golubnitschaja O. Diabetes mellitus as a risk factor for cancer: stress or viral etiology? Infect Disord Drug Targets. 2008;8:76-87.

7. Cebioglu M, Schild HH, Golubnitschaja O. Diabetes mellitus as a Risk Factor for Cancer: Is Predictive Diagnosis Possible? In: Golubnitschaja O, editor. Predictive diagnostics and personalized treatment: Dream or Reality? New York: Nova Science Publishers; 2009.

8. Frustaci A, Kajstura J, Chimenti C, et al. Myocardial cell death in human diabetes. Circ Res. 2000;87:1123-32.

9. Golubnitschaja O. Diabetes mellitus. In: Golubnitschaja O, editor. Predictive Diagnostics and Personalized Treatment: Dream or Reality? New York: Nova Science Publishers; 2009.

10. King H, Aubert RE, Herman WH. Global burden of diabetes, 1995-2025: prevalence, numerical estimates, and projections. Diabetes Care. 1998;21:1414-31.

11. Peeva V, Golubnitschaja O. Birth Asphyxia as the Most Frequent Perinatal Complication. In: Golubnitschaja O, editor. Predictive Diagnostics and Personalized Treatment: Dream or Reality? New York: Nova Science Publishers; 2009.

12. Yeghiazaryan K, Peeva V, Morelli M, et al. Potential Targets for Early Diagnosis and Neuroprotection in Asphyxiated Newborns. In: Golubnitschaja O, editor. Predictive Diagnostics and Personalized Treatment: Dream or Reality? New York: Nova Science Publishers; 2009.

13. Dunlop BW, Sternberg M, Phillips LS, Andersen J, Duncan E. Disturbed glucose metabolism among patients taking olanzapine and typical antipsychotics. Psychopharmacol Bull. 2003;37 (3):99-117.

14. Groom MJ, Scerif G, Liddle PF et al. Effects of Motivation and Medication on Electrophysiological Markers of Response Inhibition in Children with Attention-Deficit/Hyperactivity Disorder. Biol Psychiatry, PMID: 19914599. 2009.

15. Cohen D. Atypical antipsychotics and new onset diabetes mellitus. An overview of the literature Pharmacopsychiatry. 2004;37(1):1-11.

16. Starrenburg FC, Bogers JP. How can antipsychotics cause Diabetes Mellitus? Insights based on receptor-binding profiles, humoral factors and transporter proteins. Eur Psychiatry. 2009;24 (3):164-70.

17. Nakano T, Ito H. Epidemiology of diabetes mellitus in old age in Japan. Diabetes Res Clin Pract. 2007;1:76-81.

18. du Prel JB, Icks A, Grabert M, et al. Socioeconomic conditions and type 1 diabetes in childhood in North Rhine-Westphalia, Germany. Diabetologia. 2007;50:720-8.

19. Płaczkiewicz-Jankowska E, Szybiński Z, Huszno B. Environmental factors in the development of type 1 diabetes - a new insight. Prz Lek. 2007;64:175-9.

20. Golubnitschaja O. Cell cycle checkpoints: the role and evaluation for early diagnosis of senescence, cardiovascular, cancer, and neurodegenerative diseases. Amino Acids. 2007;32:359-71.

21. Niwa Y, Ishimoto K, Kanoh T. Induction of superoxide dismutase in leukocytes by paraquat: correlation with age and possible predictor of longevity. Blood. 1990;76:835-41.

22. Koehn J, Turhani D, Krapfenbauer K. Potential Predictive Biomarkers for the Dysfunction of B-cells in Type 2 Diabetes. 
In: Golubnitschaja O, editor. Predictive Diagnostics and Personalized Treatment: Dream or Reality? New York: Nova Science Publishers; 2009.

23. Koehn J, Krapfenbauer K. Advanced proteomics procedure as a detection tool for predictive screening in type 2 pre-Diabetes. The EPMA-Journal 1. 2010.

24. Lesgards JF, Durand P, Lassarre M, et al. Assessment of lifestyle effects on the overall antioxidant capacity of healthy subjects. Environ Health Perspect. 2002;110:479-86.

25. Harrison PT. Application of gene therapy in diabetes care. Infect Disord Drug Targets. 2008;8:129-33.

26. Kowluru RA, Chan PS. Oxidative stress and diabetic retinopathy. Exp Diabetes Res. 2007;2007:43603.

27. George B, Cebioglu M, Yeghiazaryan K. Inadequate diabetes care: global figures cry for preventive measures and personalized treatment. The EPMA-Journal 1. 2010.

28. Josifova T, Schneider U, Henrich PB, et al. Eye disorders in diabetes: potential drug targets. Infect Disord Drug Targets. 2008;8:70-5.

29. Sugimoto K, Yasujima M, Yagihashi S. Role of advanced glycation end products in diabetic neuropathy. Curr Pharm Des. 2008;14(10):953-61.

30. Said G, Baudoin D, Toyooka K. Sensory loss, pains, motor deficit and axonal regeneration in length-dependent diabetic polyneuropathy. J Neurol. 2008;255(11):1693-702.

31. Obrosova IG. Diabetic painful and insensate neuropathy: pathogenesis and potential treatments. Neurotherapeutics. 2009;6 (4):638-47.

32. Bailey CC, Sparrow JM. Co-morbidity in patients with sightthreatening diabetic retinopathy. Eye (Lond). 2001;15(Pt 6):71922 .

33. Kapalla M, Yeghiazaryan K, Hricová M, et al. Combined analysis of biochemical parameters in serum and differential gene expression in circulating leukocytes may serve as an ex vivo monitoring system to estimate risk factors for complications in Diabetes mellitus. Amino Acids. 2005;28:221-7.

34. Javadi MA, Katibeh M, Rafati N, et al. Prevalence of diabetic retinopathy in Tehran province: a population-based study. BMC Ophthalmol. 2009;16:9-12.

35. Mozaffari MS, Abdelsayed R, Schaffer SW. Diabetic Complications: Pathogenetic Mechanisms and Prognostic Indicators. In: Golubnitschaja O, editor. Predictive Diagnostics and Personalized Treatment: Dream or Reality? New York: Nova Science Publishers; 2009.

36. Yeghiazaryan K, Bauriedel G, Schild HH, et al. Prediction of degeneration of native and bioprosthetic aortic valves: issuerelated particularities of diabetes mellitus. Infect Disord Drug Targets. 2008;8:88-99.

37. Handel AE, Handunnetthi L, Ebers GC, Ramagopalan SV. Type 1 diabetes mellitus and multiple sclerosis: common etiological features. Nat Rev Endocrinol. 2009;5(12):655-64.

38. Silva KC, Rosales MA, Biswas SK, et al. Diabetic retinal neurodegeneration is associated with mitochondrial oxidative stress and is improved by an angiotensin receptor blocker in a model combining hypertension and diabetes. Diabetes. 2009;58 (6):1382-90.

39. Finsterer J. Treatment of mitochondrial disorders. Eur J Paediatr Neurol, PMID: 19692274. 2009.

40. Moroz N, Tong M, Longato L, et al. Limited Alzheimer-type neurodegeneration in experimental obesity and type 2 diabetes mellitus. Alzheimers Dis. 2008;15(1):29-44.

41. de la Monte SM, Wands JR. Alzheimer's disease is type 3 diabetes-evidence reviewed. J Diabetes Sci Technol. 2008;2 (6):1101-13.

42. de la Monte SM, Longato L, Tong M, et al. Insulin resistance and neurodegeneration: roles of obesity, type 2 diabetes mellitus and non-alcoholic steatohepatitis. Curr Opin Investig Drugs. 2009;10 (10):1049-60.

43. Tan GS, Wong TY, Fong CW, et al. Diabetes, metabolic abnormalities, and glaucoma. Arch Ophthalmol. 2009;127 (10):1354-61.

44. Cebioglu M, Schild HH, Golubnitschaja O. Cancer predisposition in Diabetics: Risk factors considered for predictive diagnostics and targeted preventive measures. The EPMA-Journal 1. 2010.

45. Schaub S, Wilkins J, Weiler T, et al. Urine protein profiling with surface-enhanced laser-desorption/ionization time-of-flight mass spectrometry. Kidney Int. 2004;65:323-32.

46. Mayr M, Mayr U, Chung YL, et al. Vascular proteomics: linking proteomic and metabolomic changes. Proteomics. 2004;4:3751-61.

47. Gahan PB. Circulating nucleic acids in plasma and serum: roles in diagnosis and prognosis in diabetes and cancer. Infect Disord Drug Targets. 2008;8:100-8.

48. Gahan PB. Circulating Nucleic Acids in Plasma and Serum: Roles in Diagnosis and Prognosis. In: Golubnitschaja O, editor. Predictive Diagnostics and Personalized Treatment: Dream or Reality? New York: Nova Science Publishers; 2009.

49. Abebe W, Mozaffari MS. Endothelial Dysfunction in Diabetes: Potential Application of Circulating Markers for Advanced Diagnostic and Prognostic Tools. The EPMA-Journal 1. 2010.

50. Al-Shabrawey M, Smith S. Prediction of Diabetic Retinopathy: Role of Oxidative Stress and Relevance of Apoptotic Biomarkers. The EPMA-Journal 1. 2010.

51. Golubnitschaja O Method for early diagnosis of proliferative diabetic retinopathy. International Patent No. 06115230.2. 2006.

52. Golubnitschaja O. Advanced Technologies for Prediction of Secondary Complications in Diabetes mellitus. In: Golubnitschaja O, editor. Predictive Diagnostics and Personalized Treatment: Dream or Reality? New York: Nova Science Publishers; 2009.

53. Josifova T, Henrich PB, Schrader W. Prevalence, Targeted Treatment and Prevention of Eye Disorders in Diabetes. In: Golubnitschaja O, editor. Predictive Diagnostics and Personalized Treatment: Dream or Reality? New York: Nova Science Publishers; 2009.

54. Josifova T, Henrich BP, Pleastina-Boryan I. Proliferative Diabetic Retinopathy: Predictive and Preventive Measures at Hypoxia Induced Retinal Changes. The EPMA-Journal 1. 2010.

55. Li Calzi S, Neu M, Shaw LC et al. Endothelial Progenitor Dysfunction in the Pathogenesis of Diabetic Retinopathy: Treatment Concept to Correct Diabetes-Associated Deficits. The EPMA-Journal 1. 2010.

56. Schoen FJ, Levy RJ. Calcification of tissue heart valve substitutes: progress toward understanding and prevention. Ann Thorac Surg. 2005;79:1072-80.

57. Golubnitschaja O, Yeghiazaryan K, Skowasch D, et al. G. p21WAF1/CIP1 and 14-3-3 sigma gene expression in degenerated aortic valves: a link between cell cycle checkpoints and calcification. Amino Acids. 2006;31:309-16.

58. Yeghiazaryan K, Skowasch D, Bauriedel G, et al. Could activated tissue remodeling be considered as early marker for progressive valve degeneration? Comparative analysis of checkpoint and ECM remodeling gene expression in native degenerating aortic valves and after bioprosthetic replacement. Amino Acids. 2007;32:109-14.

59. Yeghiazaryan K, Skowasch D, Bauriedel G, et al. Prediction of Degeneration of Native and Bioprosthetic Aortic Valves. In: Golubnitschaja O, editor. Predictive Diagnostics and Personalized Treatment: Dream or Reality? New York: Nova Science Publishers; 2009.

60. Baban B, Penberthy WT, Mozaffari MS. The potential Role of Indoleamine-2,3-Dioxygenase (IDO) as a Predictive and Therapeutic Target for Diabetes Treatment: A Mythical Truth. The EPMA-Journal 1. 2010. 
61. Elmarakby AA, Abdelsayed R, Liu JY et al. Inflammatory cytokines as predictive markers for early detection and progression of diabetic nephropathy. The EPMA-Journal 1. 2010.

62. Coelho JF, Ferreira P, Gil MH. New approaches in drug delivery systems: application for diabetes treatment. Infect Disord Drug Targets. 2008;8:119-28.

63. Coelho JFJ, Ferreira P, Fonseca AC, et al. Advanced Drug Delivery Systems in Diabetes Treatment. In: Golubnitschaja O, editor. Predictive Diagnostics and Personalized Treatment: Dream or Reality? New York: Nova Science Publishers; 2009.

64. Coelho J, Ferreira P, Alves P et al. Drug delivery systems: advanced technologies potentially applicable in personalized treatments. The EPMA-Journal 1. 2010.
65. Sena CM, Bento CF, Pereira P et al. Diabetes mellitus: new challenges and innovative therapies. The EPMA-Journal 1. 2010.

66. Zenoni S, Comi N, Fontana P. Individualized treatment of proliferative diabetic retinopathy: optimal surgical timing improves long-term outcomes. The EPMA-Journal 1. 2010.

67. Schrader WF, Josifova T. The options to minimize the surgical trauma to treat ocular diabetic complications and to improve postoperative recovery and quality of life require an individualized approach. The EPMA-Journal 1. 2010.

68. Hanes PhJ, Krishna R. Characteristics of inflammation common to both diabetes and periodontitis: are predictive diagnosis and targeted preventive measures possible? The EPMA-Journal 1. 2010 . 